\title{
Quantum technologies in diamond enabled by laser processing
}

Cite as: Appl. Phys. Lett. 120, 020502 (2022); https://doi.org/10.1063/5.0080348

Submitted: 30 November 2021 • Accepted: 29 December 2021 Published Online: 10 January 2022

Published open access through an agreement withPublished open access through an agreement with Consiglio Nazionale delle Ricerche

A. N. Giakoumaki, (D) G. Coccia, (iD) V. Bharadwaj, et al.

(1)

母QBLOX

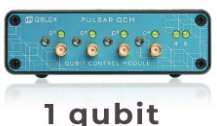

Shorten Setup Time Auto-Calibration More Qubits

Fully-integrated Quantum Control Stacks Ultrastable DC to $18.5 \mathrm{GHz}$ Synchronized $<<1$ ns Ultralow noise

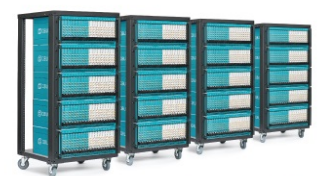

100s qubits

visit our website > 


\title{
Quantum technologies in diamond enabled by laser processing
}

\author{
Cite as: Appl. Phys. Lett. 120, 020502 (2022); doi: 10.1063/5.0080348 \\ Submitted: 30 November 2021 - Accepted: 29 December 2021 . \\ Published Online: 10 January 2022
}

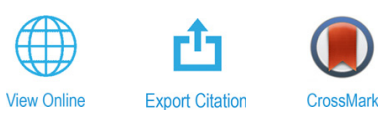

\begin{abstract}
A. N. Giakoumaki, (iD G. Coccia, ${ }^{1}$ (D) V. Bharadwaj, ${ }^{1}$ (D) J. P. Hadden, 2,3 (iD A. J. Bennett, ${ }^{3,4}$ (iD B. Sotillo, ${ }^{5}$ R. Yoshizaki, ${ }^{6}$ (D) P. Olivero, ${ }^{7}$ (D) O. Jedrkiewicz, ${ }^{8}$ (D R. Ramponi, ' iD S. M. Pietralunga, (iD M. Bollani, ${ }^{7}$ A. Bifone, ${ }^{10,11}$ P. E. Barclay, ${ }^{2}$ A. Kubanek 12,13 and S. M. Eaton
\end{abstract}

\author{
AFFILIATIONS \\ ${ }^{7}$ Istituto di Fotonica e Nanotecnologie-Consiglio Nazionale delle Ricerche (IFN-CNR) and Dipartimento di Fisica-Politecnico di \\ Milano, Piazza Leonardo da Vinci 32, Milano 20133, Italy \\ ${ }^{2}$ Institute for Quantum Science and Technology, University of Calgary, Calgary, Alberta T2N 1N4, Canada \\ ${ }^{3}$ School of Physics and Astronomy, Cardiff University, Cardiff CF24 3AA, United Kingdom \\ ${ }^{4}$ School of Engineering, Cardiff University, Cardiff CF24 3AA, United Kingdom \\ ${ }^{5}$ Department of Materials Physics-Complutense University of Madrid, Ciudad Universitaria, 28040-Madrid, Spain \\ ${ }^{6}$ Department of Mechanical Engineering, School of Engineering, University of Tokyo, Tokyo 113-8656, Japan \\ ${ }^{7}$ Department of Physics and "Nanostructured Interfaces and Surfaces" Inter-Departmental Centre, University of Torino, \\ I-10125 Torino, Italy \\ ${ }^{8}$ Istituto di Fotonica e Nanotecnologie-Consiglio Nazionale delle Ricerche (CNR-IFN), Department of Science and High Technology, \\ Università degli Studi dell'Insubria, Via Valleggio 11, 22100 Como, Italy \\ ${ }^{9}$ Istituto di Fotonica e Nanotecnologie, CNR, L-NESS, Via Anzani 42, 22100 Como, Italy \\ ${ }^{10}$ Istituto Italiano di Tecnologia, Center for Sustainable Future Technologies, via Livorno 60, 10144 Torino, Italy \\ ${ }^{11}$ University of Torino, Molecular Biology Center, via Nizza 52, 10126 Torino, Italy \\ ${ }^{12}$ Institute for Quantum Optics, Ulm University, D-89081 UIm, Germany \\ ${ }^{13}$ Center for Integrated Quantum Science and Technology (IQst), UIm University, D-89081 Ulm, Germany
}

a) Author to whom correspondence should be addressed: shane.eaton@gmail.com

\begin{abstract}
Integrated photonic circuits promise to be foundational for applications in quantum information and sensing technologies, through their ability to confine and manipulate light. A key role in such technologies may be played by spin-active quantum emitters, which can be used to store quantum information or as sensitive probes of the local environment. A leading candidate is the negatively charged nitrogen vacancy $\left(\mathrm{NV}^{-}\right)$diamond color center, whose ground spin state can be optically read out, exhibiting long $(\approx 1 \mathrm{~ms})$ coherence times at room temperature. These properties have driven research toward the integration of photonic circuits in the bulk of diamond with the development of techniques allowing fabrication of optical waveguides. In particular, femtosecond laser writing has emerged as a powerful technique, capable of writing light guiding structures with 3D configurations as well as creating NV complexes. In this Perspective, the physical mechanisms behind laser fabrication in diamond will be reviewed. The properties of waveguides, single- and ensemble-NV centers, will be analyzed, together with the possibility to combine such structures in integrated photonic devices, which can find direct application in quantum information and sensing.
\end{abstract}

(C) 2022 Author(s). All article content, except where otherwise noted, is licensed under a Creative Commons Attribution (CC BY) license (http:// creativecommons.org/licenses/by/4.0/). https://doi.org/10.1063/5.0080348

The existence of stable, fluorescent, and spin-active color centers has made diamond a promising platform for quantum photonics. In particular, its negatively charged nitrogen-vacancy $\left(\mathrm{NV}^{-}\right)$color center has unveiled long spin coherence times $(\approx 1 \mathrm{~ms})$ even at room temperature, ${ }^{1}$ making it an appealing system for quantum applications. The
$\mathrm{NV}^{-}$center, which appears intrinsically in both natural and synthetic diamond, results from the replacement of two adjacent sites of the diamond carbon lattice by a nitrogen and a vacancy. The electronic ground state of such a complex forms a spin triplet, which can be polarized under green excitation, and exhibits a zero-phonon line 
(ZPL) at a $637 \mathrm{~nm}$ wavelength. ${ }^{2}$ Optical spin readout can be achieved through measurement of the fluorescence signal due to spin dependent non-radiative decay paths, which make one spin state brighter than the others. ${ }^{3}$ Moreover, $\mathrm{NV}^{-}$centers in diamond can be called into play for sensing applications, being sensitive to magnetic and electric fields through the Zeeman and Stark effects, respectively. The measurement of strain, ${ }^{4}$ temperature, ${ }^{5}$ and magnetic fields ${ }^{6-8}$ at the nanoscale with high sensitivity has already been demonstrated, with compelling applications in the biological sciences ${ }^{9,10}$ as well as in other fields. ${ }^{11,12}$

The implementation of such systems requires the ability to create $\mathrm{NV}^{-}$centers in a controlled way, with high precision and a desired density, from single color center to high density ensembles. Additionally, it is desirable to fabricate optical waveguides, to enable strong light-matter interaction and to link and spatially address $\mathrm{NV}^{-}$ centers. Indeed, this is a very challenging task due to diamond's extreme hardness and chemical resistance. Conventionally, this has been addressed through plasma etching, ${ }^{13,14}$ ion implantation, ${ }^{15}$ or ion beam-assisted liftoff, ${ }^{16}$ all invasive techniques that inevitably degrade the quality of the diamond surface and consequently device performance, as summarized in Table I. A solution to such problems has recently been found in femtosecond laser writing of photonic structures, ${ }^{17,18}$ benefiting from the localized bulk modification due to nonlinear absorption of focused ultrashort pulses. ${ }^{19,20}$ In this way, direct writing of 3D integrated optical circuit has been demonstrated, ${ }^{21,22}$ with the possibility to create $\mathrm{NV}^{-}$color centers in a controlled manner, while retaining their high-optical and spin coherence. ${ }^{23}$ In this Perspective, first, the physical mechanism behind the fabrication of optical waveguides in bulk diamond will be explained. Subsequently, the integration of such waveguides with $\mathrm{NV}^{-}$ centers will be analyzed, and in particular, results on the creation and interaction of single-color centers within optical waveguides will be presented. Finally, the creation of ensembles of $\mathrm{NV}^{-}$centers with a desired density will be shown, together with a proof-of-concept application given by a sensing device capable of magnetic field and temperature sensing. An outlook of the Perspectives of the technique will underline the future developments for the described devices in terms of complexity and functionality, toward a fully integrated laser written photonic device in diamond.

The use of ultrashort laser pulses to modify the internal structure of transparent materials has become an established technique over the last 20 years. The material modification due to focused femtosecond lasers pulses can assume different properties depending on the absorbed energy at the focal volume, ${ }^{35-38}$ in addition to other properties such as translation velocity, polarization, repetition rate, wavelength, beam shape, focusing condition, and pulse duration. In

TABLE I. Comparison of femtosecond laser writing with other techniques for waveguides fabrication and $\mathrm{NV}^{-}$center formation.

\begin{tabular}{|c|c|c|}
\hline & Femtosecond laser writing & Other techniques \\
\hline Waveguides & $\begin{array}{l}\text { - Easy single step, 3D fabrication }{ }^{17,18} \\
\text { - Type II fabrication of waveguides with stress-induced guiding } \\
\text { between damaged laser-formed tracks }{ }^{17} \\
\text { - Single mode guiding by tailoring core size for specific wave- } \\
\text { lengths }(13 \mu \mathrm{m} @ 635 \mathrm{~nm}, 19 \mu \mathrm{m} @ 1550 \mathrm{~nm}, 30 \mu \mathrm{m} @ 2.4 \mu \mathrm{m} \text {, } \\
60 \mu \mathrm{m} @ 4.8 \mu \mathrm{m})^{24,25} \\
\text { - Easy coupling with optical fibers } \\
\text { - Variable depth in the bulk of diamond } \\
\text { - from } 12 \mu \mathrm{m} \text { to } \approx 200 \mu \mathrm{m}^{24} \\
\text { - Not possible to fabricate surface photonics for quantum sensing } \\
\text { - Modest refractive index increase between waveguide region and } \\
\text { bulk }\left(\approx 10^{-3}\right)^{18} \\
\text { - Not possible to write nm dimension structures } \\
\text { - Only TM polarization guiding possible }{ }^{17} \text { unless depressed clad- } \\
\text { ding geometry adopted }\end{array}$ & $\begin{array}{l}\text { - possibility to create nanocavities and } \\
\text { resonators } \\
\text { - hard to couple with optical fibers } \\
\text { - Superficial modifications }<50 \mu \text { mepth }^{15} \\
\text { - Demonstrated complex structures through } \\
\text { Nanolithography and RIE }{ }^{28,30} \\
\text { - Different and complex fabrication setup for } \\
\text { each application }\end{array}$ \\
\hline NV centers & $\begin{array}{l}\text { - Deterministic single } \mathrm{NV}^{-} \text {formation with realtime confocal fluo- } \\
\text { rescence feedback } \\
\text { - Localized within } 600 \mathrm{~nm} \text { in the focus }{ }^{26} \\
\text { - Possibility to form ensembles of } \mathrm{NV}^{-} \text {with controlled density } \\
\text { - Demonstrated integration with other laser written components }\end{array}$ & $\begin{array}{l}\text { - Main alternative: Ion beam implantation } \\
\text { - Randomly distributed single/double } \mathrm{NVs}^{-} \\
\text {within the focal volume }{ }^{31} \\
\text { - Localized within tens of nm laterally }{ }^{32} \\
\text { - Ensembles of } \mathrm{NVs}^{-} \text {with controlled density } \\
\text { - Damage of the crystal along the focused beam } \\
\text { direction } \\
\left.\text { - Exotic color centers (ex. } \mathrm{SiV}^{-}\right)^{34}\end{array}$ \\
\hline
\end{tabular}


crystals, femtosecond laser irradiation generally produces a decrease in refractive index at the focal volume, caused by the creation of lattice disorder, leading to a lower density of the lattice. It has been demonstrated that it is still possible to achieve guiding of light in the bulk of crystals if two parallel lines separated by several micrometers are fabricated, confining the optical mode between these barriers, where the refractive index is higher (type II modification). ${ }^{17,38}$ In Fig. 1(a), it is shown how the TM mode of a red CW-laser can be confined within two laser-written tracks in diamond. Sotillo et al. showed how the guiding of the TM optical modes is related to the structural modification of the material. ${ }^{39}$ In Fig. 1(b), a map of the refractive index change in proximity to the laser-written tracks shows a reduction of the refractive index inside the tracks and at their apex, while a relative refractive index increase is produced in between the two parallel lines, where $\Delta n$ was calculated to be about $3 \times 10^{-3} \cdot \cdot^{40-42}$ From an analysis of the Raman peak shifts and change of intensity of the Raman signal at the modification, ${ }^{43,44}$ a map of the stress components along two perpendicular directions of the crystal lattice has been developed [Figs. 1(c) and 1(d)]. The results show amorphization and the transformation of $\mathrm{sp}^{3}$-diamond bonding into $\mathrm{sp}^{2}$ bonding in the laser written lines. As a consequence of this density reduction, stress is exerted between the lines, which depends on the crystal lattice direction: along the horizontal direction, the stress is compressive [Fig. 1(c)], whereas in the vertical direction, a shear tensile stress is established [Fig. 1(d)]. This explains why only the TM mode can be guided by the type II waveguide in diamond, ${ }^{17}$ being parallel to the tensile stress component that experiences a refractive index increase, while the TE mode lies along the compressive stress component where it experiences a refractive index decrease.

(a)
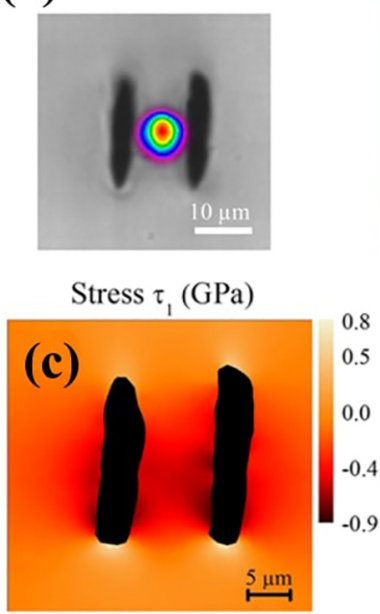

FIG. 1. (a) Optical microscopy image of a type II waveguide in diamond with overlaid the measured optical TM mode confined between the tracks. (b) Map of the refractive index profile. (c) and (d) Maps of the horizontal and vertical components of induced stresses (named as $\tau_{1}$ and $\tau_{2}$, respectively) in the waveguide from the Raman peak shift. Adapted with permission from Sotillo et al., Appl. Phys. Lett. 112, 031109 (2018). Copyright 2018 American Institute of Physics.
For practical implementation of quantum systems or high-spatial-resolution sensing devices, it is not sufficient to confine and guide light through waveguides. Deterministic placement of $\mathrm{NV}^{-}$centers is also necessary. In 2013, it was shown how femtosecond laser pulses focused slightly above the surface of optical grade diamond can induce the formation of $\mathrm{NV}^{-}$centers through surface ablation. ${ }^{45}$ A further step was taken by Chen et al., demonstrating on-demand and highquality single NVs written in the bulk of electronic grade diamond, with a nitrogen concentration of $<5 \mathrm{ppb}$, using femtosecond laser writing with a spatial light modulator (SLM) to correct for aberrations, followed by high-temperature annealing. ${ }^{21}$ This is achieved by the formation of vacancies within the focal volume when femtosecond laser pulses with energies below the amorphization threshold are focused in the bulk of diamond. The high temperature annealing mobilizes the vacancies, which eventually are captured by substitutional nitrogen impurities to form NV centers. ${ }^{22,26}$

Yurgens et al. ${ }^{46}$ recently proposed an alternative focusing method relying on a solid-immersion lens (SIL), which enabled laser writing at pulses energies as low as $5.8 \mathrm{~nJ}$ per pulse, where lattice vacancies were created by tunneling breakdown rather than multiphoton ionization. These low pulse energies are fivefold lower than the first report by Chen et al. and also offer the advantage of using a lower-cost femtosecond laser oscillator instead of an amplified system. Yurgens et al. were able to demonstrate single NVs as shallow as $1 \mu \mathrm{m}$ with the SIL method.

The spin coherence times of laser-induced NVs are shown to be comparable with those implanted by ion beams. ${ }^{26}$ The development of this powerful technique overcame near surface fabrication limitations related to previous works, also providing an alternative to ion implantation, ${ }^{27,39,47}$ the most common technique for color center creation, but which can lead to significant damage of the diamond lattice.

A challenging task for the development of integrated photonic circuits is related to the integration of the color centers with the optical waveguides. ${ }^{48-50}$ Recently, the possibility to write single $\mathrm{NV}^{-}$centers in electronic grade diamond coupled to an optical waveguide has been demonstrated. ${ }^{51}$ The fabrication consisted of three steps. First, a Type II waveguide was written in a bulk diamond at a depth of $25 \mu \mathrm{m}$ using the second harmonic of a regeneratively amplified Yb:KGW system (Pharos, Light Conversion), with a $515 \mathrm{~nm}$ wavelength, at a repetition rate of $500 \mathrm{kHz}$ with a $0.6 \mathrm{~nJ}$ pulse energy and $0.5 \mathrm{~mm} / \mathrm{s}$ scanning speed. A separation of $13 \mu \mathrm{m}$ between the two laser-written tracks was used to optimize single mode propagation of $637 \mathrm{~nm}$ radiation corresponding to the ZPL of the $\mathrm{NV}^{-}$center emission. Second, the same femtosecond laser system was used for single-pulse exposures within the waveguide to induce vacancies in the diamond lattice. To study the reproducibility of the experiment, five identical single-pulse exposures separated by $20 \mu \mathrm{m}$ were performed at the center of the tracks defining the optical waveguide, with an energy of $28 \mathrm{~nJ}$ per pulse. Next, the sample was annealed for 3 hours at $1000{ }^{\circ} \mathrm{C}$ in a nitrogen atmosphere to induce the formation of the $\mathrm{NV}^{-}$centers and anneal out other detrimental vacancy complexes. ${ }^{52}$ Importantly, this did not compromise the previously written waveguide, in contrast to studies in other materials where the high temperatures treatment caused the lattice to recover its initial structure. ${ }^{53}$ Figure 2(a) shows an overhead optical microscope image of the waveguide- $\mathrm{NV}^{-}$optical device. The fabricated device was characterized using a $532 \mathrm{~nm}$ excitation laser focused either through a microscope objective or through single-mode fiber 
(a)

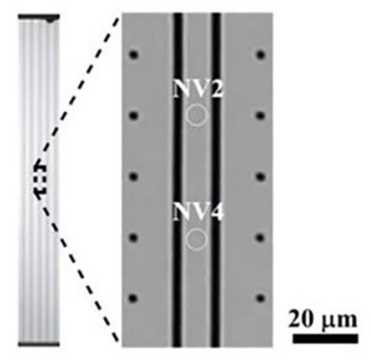

(c)

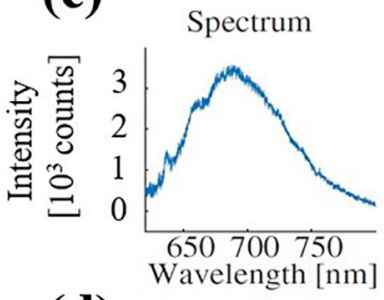

(d)

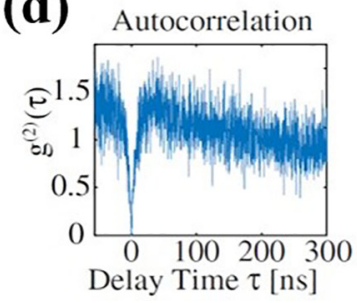

(b)

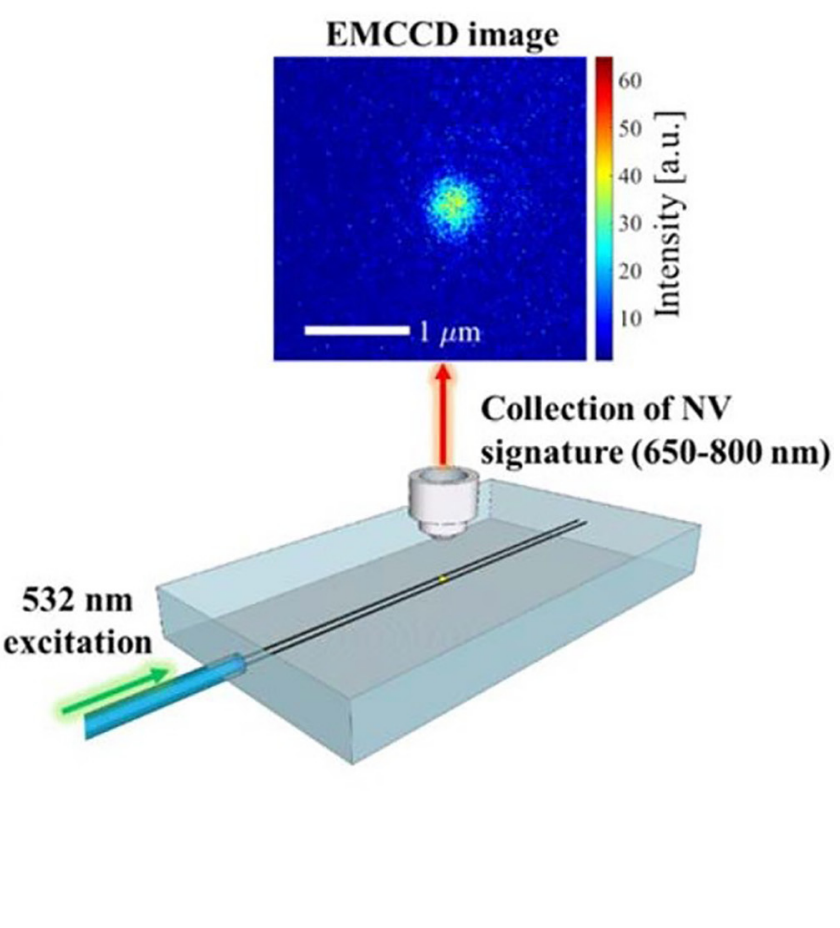

FIG. 2. (a) Overhead optical microscope image of the $2 \mathrm{~mm}$ long waveguide, with a zoom-in of the central $80 \mu \mathrm{m}$ containing the NV centers 2 and 4 (NV2 and NV4). (b) Schematic of the experimental setup for the characterization of the color centers and their coupling with the waveguide. (c) PL spectrum displaying NV ZPL at $637 \mathrm{~nm}$. (d) NV intensity autocorrelation confirming single-photon emission. Adapted with permission from Hadden et al., Opt. Lett. 43, 3586-3589 (2018). Copyright 2018 Optical Society of America/Optica.

that was back-coupled to the waveguide. The emitted light was then detected from the other end of the waveguide or from overhead as shown in Fig. 2(b). The single NV formation probability, including trials in other waveguides, was estimated as $(31 \pm 9) \%$, consistent with the Salter group's initial work. The NV placement accuracy was estimated to be $\sim 1 \mu \mathrm{m}$, larger than the $\sim 600 \mathrm{~nm}$ value that was previously reported, due to the larger focal volume provided by the femtosecond writing setup. Figure 2(c) shows the emission spectrum from one of the $\mathrm{NV}^{-}$centers at room temperature, showing the characteristic ZPL at $637 \mathrm{~nm}$ and the broadband phonon sideband (PSB), while Fig. 2(d) shows the second-order intensity autocorrelation chronogram $\mathrm{g}^{2}(\tau)$, confirming single-photon emission. Laser written waveguides allowing excitation and fluorescence collection from precisely located and single-photon $\mathrm{NV}^{-}$emitters resulted in a simple device opening the way toward more complex systems, necessary in the field of quantum information.

Room temperature quantum sensing is one of the main applications of color centers, due to the susceptibility of their spin states to electric and magnetic fields. ${ }^{8,10,54-57}$ Single $\mathrm{NV}^{-}$centers have been used to show nanoscale spatial resolution surpassing the resolution of the optical fluorescence microscopes used for measurement, with the intrinsic low sensitivity related to the use of a single $\mathrm{NV}^{-}$center increased by averaging over many measurements. When nanoscale spatial resolution is not required, the sensitivity or the measurement speed can be increased through the use of $\mathrm{NV}^{-}$center ensembles, as already shown through high density $\mathrm{NV}^{-}$centers in bulk diamond. ${ }^{55}$ It has been demonstrated that the sensitivity of such devices scales as $\sqrt{M},{ }^{58}$ where $M$ is the number of color centers in the ensemble. This can be achieved by either increasing volume of $\mathrm{NV}^{-}$centers or the density of sampled $\mathrm{NV}^{-}$centers while not compromising on their spin coherence time. Ensembles with several $\mathrm{NV}^{-}$densities in CVD and HPHT grown diamond with higher intrinsic nitrogen content have been studied ${ }^{59-62}$ to optimize the sensitivity without a reduction of the spin coherence time of the system. Clevenson et al. identified an optimal $\mathrm{NV}^{-}$density of $2 \times 10^{16} \mathrm{~cm}^{-3}(0.1 \mathrm{ppm}){ }^{63}$ In agreement with previous studies, high density $\mathrm{NV}^{-}$center ensembles have been written in HPHT diamond through single static exposures followed by thermal annealing, and their integration within a waveguide has been characterized. ${ }^{22}$ Arrays of "empty" waveguides together with "static exposure" ones (containing arrays of nine single 100-nJ static exposures uniaxially separated by $2 \mu \mathrm{m}$ ) were written in HPHT diamond at a depth of $18 \mu \mathrm{m}$. During annealing, $\mathrm{NV}^{-}$s are generated throughout the waveguide mode due to the diffusion of vacancies from the modification lines, as can be seen from the confocal microscope images of Figs. 3(a) and 3(b). Figure 3(c) shows PL spectra acquired after annealing from the "empty," "static exposure" waveguides and from a bright spot corresponding to a static exposure in the waveguide. In all three cases, the characteristic ZPL of the $\mathrm{NV}^{-}$center can be identified, 
(a)
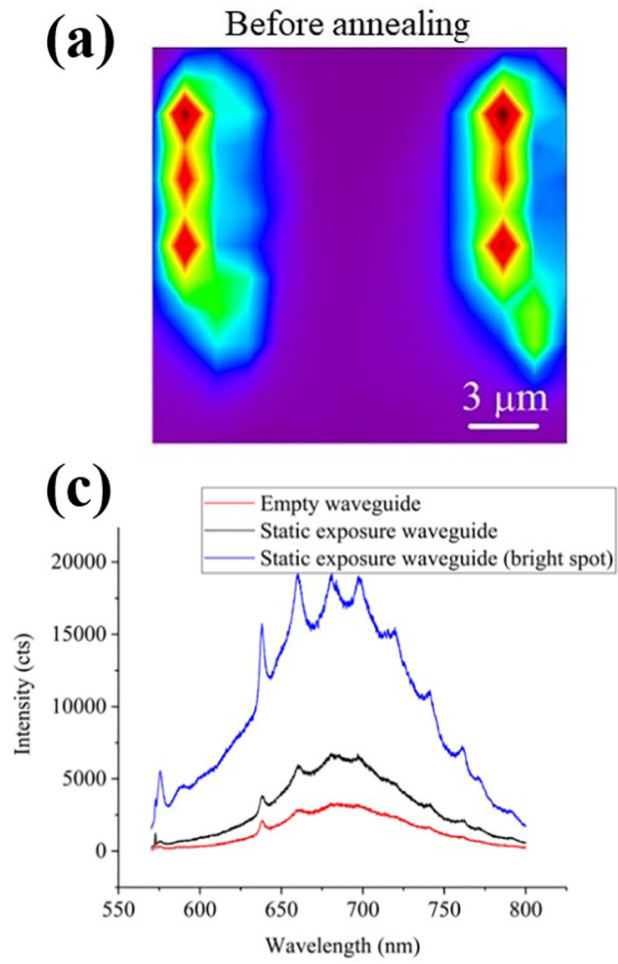

(b)

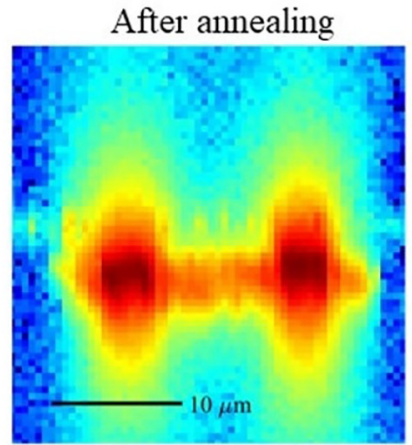

(d)

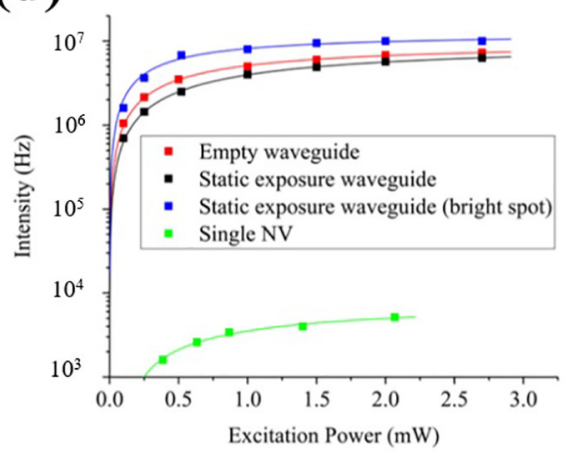

FIG. 3. Photoluminescence confocal cross section of "empty" waveguide and modification lines written in HPHT sample before (a) and after (b) annealing. PL spectra (c) and Power dependent PL (d) from empty waveguide, "static exposure" waveguide, and from a bright spot corresponding to a static exposure. Adapted with permission from Eaton et al., Adv. Quantum Technol. 2, 1900006 (2019). Copyright 2019 Wiley$\mathrm{VCH} \mathrm{GmbH}$. confirming $\mathrm{NV}^{-}$creation. In addition, the static exposure waveguides show enhanced signal as well as additional features, for example, the strong contribution of a line at $575 \mathrm{~nm}$ corresponding to the neutral $\mathrm{NV}^{0}$ ZPL. It was possible to estimate the density of the ensembles from the power dependent PL saturation measurements of the considered waveguides compared to the signal collected by a single $\mathrm{NV}^{-}$center in another sample [Fig. 3(d)], resulting in a $1.1 \times 10^{15} \mathrm{~cm}^{-3}$ value (corresponding to $6 \mathrm{ppb}$ ) in the "empty" waveguide, and up to $1.4 \times 10^{15} \mathrm{~cm}^{-3}(8 \mathrm{ppb})$ in the "static exposure" one. It has been estimated that these densities would permit a magnetic field sensitivity of $1.5 \mathrm{nT} \mathrm{Hz}^{-1 / 2}$ or an electric field sensitivity of $2.4 \mathrm{~V} \mathrm{~cm}^{-1} \mathrm{~Hz}^{-1 / 2}$, which are comparable to those allowed by current state-of-the-art systems ${ }^{58,62}$ and which could be further improved by optimization of the laser fabrication parameters.

A first demonstration of a device combining laser written waveguides arrays with shallow ion implanted $\mathrm{NV}^{-}$center ensembles for temperature and magnetic field sensing has recently been reported by Hoese et al. ${ }^{64}$ Such a system combines the sensing capabilities of $\mathrm{NV}^{-}$ ensembles with the photon routing properties of optical waveguides, separating the optical access to the NV centers from the object to be sensed and allowing noninvasive field detection from biological and chemical samples [Fig. 4(a)]. This is an advantage compared to confocal microscopy as the waveguide platform is fully integrated and can be scaled to many channels, which are laterally separated. The device was fabricated by laser-writing type II optical waveguides in depths ranging from 5 to $25 \mu \mathrm{m}$ below the diamond surface, followed by shallow implantation of nitrogen ions into the front facet of the waveguides, which resulted in $\mathrm{NV}^{-}$-ensemble creation after annealing at $1000^{\circ} \mathrm{C} . \mathrm{NV}^{-}$center spin state measurement was performed via room temperature optically detected magnetic resonance (ODMR). ${ }^{65}$ Offresonant $532 \mathrm{~nm}$ laser excitation and $\mathrm{NV}^{-}$center fluorescence collection coupled through a $0.25 \mathrm{NA}$ objective from the end facet of the waveguide are fully compatible with the placement of biological or chemical samples. A confocal scan of the end facet of the sample in Fig. 4(b) shows a clear waveguide mode corresponding to remote $\mathrm{NV}^{-}$excitation and fluorescence detection. A comparison of the spectra in Fig. 4(c) confirms that the waveguide-assisted spectrum resembles that of the directly collected free space fluoresce from the $\mathrm{NV}^{-}$ensemble. The ODMR sensing performance of an ensemble was studied by monitoring the fluorescence while applying a swept microwave (MW) field to the sample and varying external magnetic field. Upon application of a magnetic field, an increased splitting between the two characteristic dips in fluorescence is observed when measured both in the free space and waveguide-assisted configurations [Fig. 4(d)]. ${ }^{39,54,66}$ Such a change in the splitting can be directly linked to the applied magnetic field, ${ }^{67,68}$ which is detected with a precision better than $6 \mu \mathrm{T}$ with this device configuration. Furthermore, in a typical 30 min CW-ODMR measurement, sensitivities up to $36 \mu \mathrm{T} \mathrm{Hz}{ }^{-1 / 2}$ (for confocal detection) and $62 \mu \mathrm{T} \mathrm{Hz}^{-1 / 2}$ (for detection after waveguide transmission) have been reached, which can be improved when using the lock-in, ${ }^{69}$ pulsed ODMR or Ramsey spectroscopy techniques. ${ }^{70}$ Finally, the device works reliably over more than a 20 -hour period with a minimal measurement time below $40 \mathrm{~s}$ and has been shown to be working also as a temperature sensor, by exploiting the temperature-dependence of the zero-field splitting (ZFS).

Despite the first implementations of simple, proof-of-concept devices revealing the possibilities of laser written photonics in diamond, there is still large room for improvement for the fabrication of 
(a)

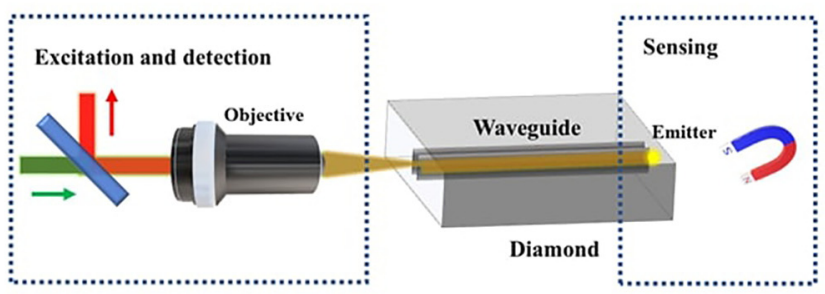

(b)

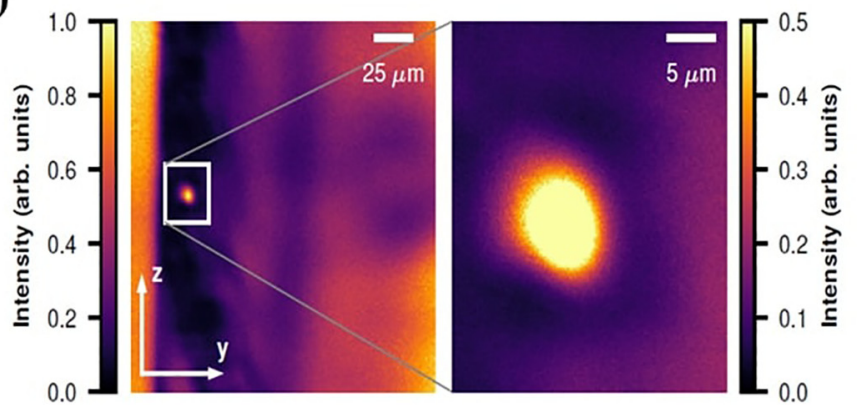

(c)

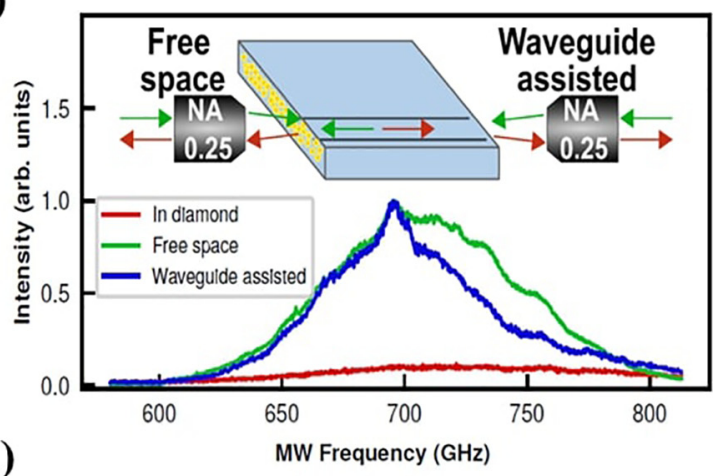

(d)

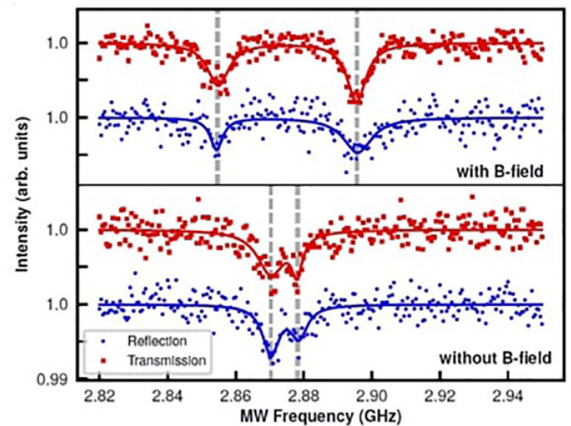

FIG. 4. (a) Schematic showing spatially separated detection and sensing region of the device. (b) Confocal scan of the end facet of the sample with a low-NA objective and a zoomed-in view of the waveguide mode. (c) Comparison of the PL spectra in transmission (blue, waveguide assisted) and reflection (green, free space) of the waveguide mode as well as the background signal (red, in diamond) given by the transmission outside the waveguide mode. (d) ODMR spectrum at zero magnetic field and with applied magnetic field detected in confocal configuration (blue) and in transmission through the waveguide (red). Adapted with permission from Hoese et al., Phys. Rev. Appl. 15, 054059 (2021). Copyright 2021 American Physical Society.

diamond-based devices in terms of waveguide quality, color center formation, and integration between different techniques, which will allow the development of more complex photonic integrated circuits. On the one side, the relatively large mode field diameter of laser-written waveguides inevitably leads to a low interaction strength on the single emitter level resulting in a rather low cooperativity. However, utilizing defect center creation on the waveguides side-facets enables to operate in a regime of high color center density within the field maximum of the waveguide mode. With such high-density ensembles of NV centers, future quantum sensing tasks with high sensitivity come into reach, as already proved in the past sections. Furthermore, the device architecture facilitates the combination A resulting in a strong focus and a strong interaction strength on the single emitter level. Consequently, a large single-emitter cooperativity will give an opportunity to feed individual waveguides with single photons and with high efficiency, therefore paving the way toward on-chip operation on the single quantum level. In addition, techniques enabling a better control of the creation of $\mathrm{NV}^{-}$centers are being tailored, where one could, for example, use laser pulses to increase the pool of electron donors $\left(\mathrm{NV}^{-}\right)$by changing the charge state of neutral $\mathrm{NV}^{0} \mathrm{~s}$, as already demonstrated in diamond bombarded with ions and rich in defects. Quantum photonics in diamond will also benefit by employing laser writing to integrate other vacancy-related color centers in diamond. Group IV centers such as the silicon vacancy ( $\mathrm{SiV}, \mathrm{ZPL}$ at $738 \mathrm{~nm}$ ) have superior spectral properties due to their inversion symmetry making them promising as a single photon source for quantum photonics. $^{72}$

Rong et al. recently demonstrated to generate single negatively charged silicon vacancy color centers by focusing a femtosecond laser on top of a high-purity diamond coated with a layer of $\mathrm{Si}$ nanoball. ${ }^{73}$ However, a hybrid method involving ion implantation into femtosecond laser written photonics could provide even higher quality SiV quantum emitters in diamond for quantum optics applications ${ }^{74}$ [Fig. 5(a)]. Through a similar approach, devices based on other group IV color centers are being explored. These centers display a similar defect structure and opto-physical properties as those of the SiVs, the most promising being the germanium-related (GeV, ZPL at $602 \mathrm{~nm})$, tin-related $(\mathrm{SnV}, \mathrm{ZPL}$ at $620 \mathrm{~nm})$, and lead-related $(\mathrm{PbV}, \mathrm{ZPL}$ at $520 \mathrm{~nm}$ ) color centers. ${ }^{75-81}$ In addition to diamond, other material platforms show promise for laser writing of quantum emitters such as hexagonal boron nitride ${ }^{82}$ and silicon carbide. ${ }^{83}$ The reader is pointed to the excellent review article by Gao et al., which provides an overview of laser writing of quantum emitters in crystals. ${ }^{84}$

Through the same fabrication technique, microfluidic components ${ }^{23,85}$ will be produced and interfaced with complex photonics with nearby NV centers to enable efficient quantum sensing of biomolecules under flow conditions, as shown in Fig. 5(b). The biocompatibility of diamond, the use of all-optical methods, and the strong fluorescence of dense NVs are ideally suited for implementing lab-onchip diagnostics. For example, paramagnetic reactive oxygen species 


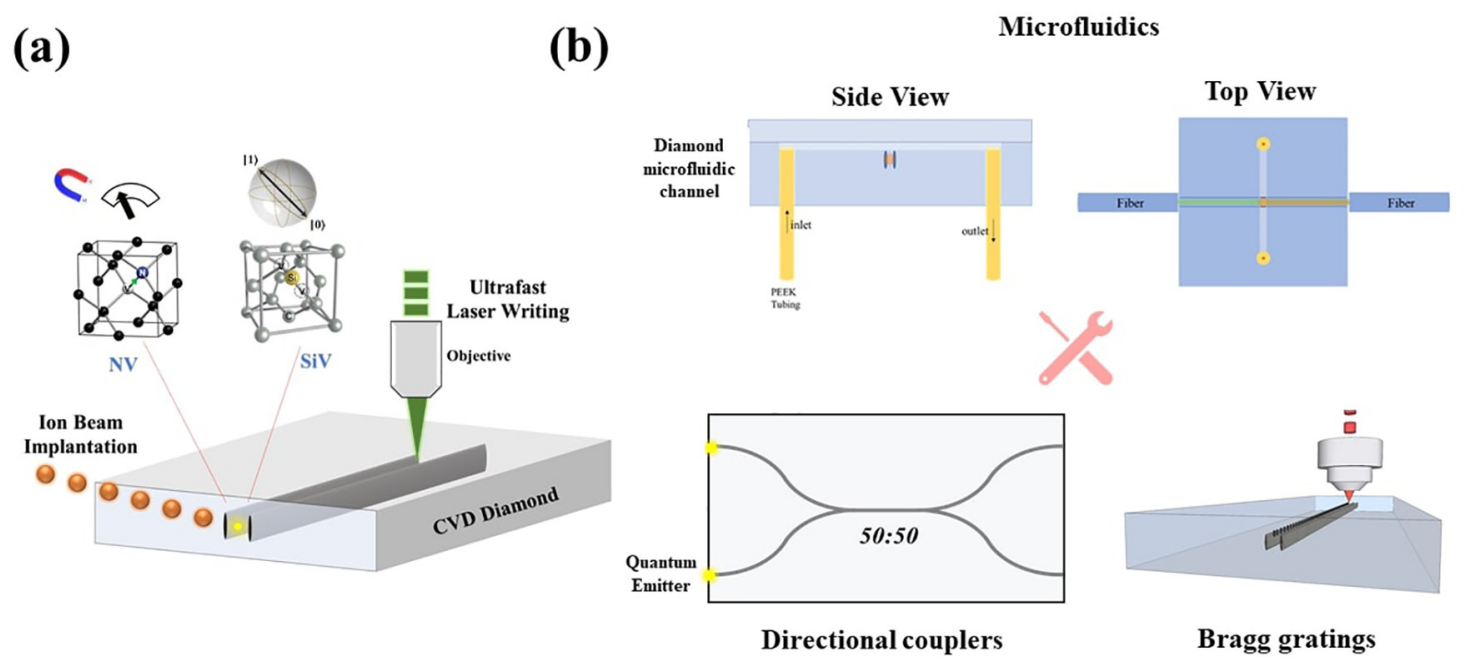

FIG. 5. (a) Schematic overview of employing lon beam implantation and ultrafast laser writing in diamond to create integrated photonic networks using NVs for magnetometry and SiVs for quantum information. (b) Schematic overview of engineering laser writing strategy to create integrated microfluidics, directional couplers, and Bragg gratings.

and free radicals are involved in cell signaling, in the aging process and in pathological states. However, detection with conventional electron spin resonance does not afford sufficient sensitivity to study their synthesis and trafficking in a physiologically relevant environment. The Bifone group has demonstrated that coupled charge and spin dynamics in dense ensembles ( $>5 \mathrm{ppm}$ ) of NVs are strongly affected by interaction with electron spins, ${ }^{86}$ enabling the detection of paramagnetic species with ultrahigh sensitivity through relaxation measurements. ${ }^{87}$ Indeed, the large number of detected photons from such dense ensembles of $\mathrm{NV}^{-}$can dramatically reduce acquisition time, down to $10 \mathrm{~ms}$, for the detection of a few tens of unpaired electron spins per $1000 \mathrm{~nm}^{2}$ of diamond surface. Importantly, incoherent detection of the evolution of polarized NV ensembles can be performed without microwaves and at ambient conditions, thus paving the way to applications in biological samples. Femtosecond laser writing shows great potential for realizing specific patterns of dense NVs in a diamond microfluidic chip, enabling all-optical detection of paramagnetic species.

On the topic of the functionalization of the devices and furthering the already demonstrated Y-splitters in diamond using laser writing, ${ }^{21}$ realization of $3 \mathrm{~dB}$ directional couplers in diamond integrated with quantum emitters will allow the formation of complex photonic networks for single photon interference and Hong-Ou-Mandel experiments. ${ }^{88}$ Moreover, wavelength-selective reflective elements such as Bragg gratings at the visible wavelengths can be integrated in waveguide devices to improve the excitation and collection efficiencies from the quantum emitters ${ }^{21}$ [Fig. 5(b)].

Indeed, laser writing has emerged as a promising fabrication platform for diamond integrated quantum photonics. From the first results of laser written diamond photonic networks in the year $2016,{ }^{17,18}$ until now, more than 30 high-impact publications employing laser writing to form photonic networks, quantum emitters, and microfluidics by various research groups from around the world have endorsed the capabilities of laser writing for the future of quantum technologies. Currently, one of the main limitations of femtosecond laser writing in diamond with respect to other techniques is due the field's immaturity only over the past five years. In comparison, there has been significantly more research on ion beam lithography in diamond, a method that was pioneered in $1970 \mathrm{~s}^{89}$ (i.e., when ion beam irradiation was already a well-established technique in the semiconductor industry), and subsequently was successfully implemented for the fabrication of graphitic structures in this material for different applications (Ohmic contacts, ${ }^{90}$ color center electrical stimulation, ${ }^{91}$ particle detection, ${ }^{92}$ metallo-dielectric photonics crystals, ${ }^{93}$ refractive index modulation, ${ }^{94}$ biosensing, ${ }^{95}$ etc.). However, a rapid development of new techniques and protocols in the framework of the laser fabrication of photonics circuits in diamond is justified by the numerous advantages of such a technique. With substantial advantages with respect to ion-irradiation based techniques, ${ }^{15}$ it results as the most convenient and versatile technique for the bulk fabrication of waveguiding structures, making possible the reliable formation of advanced 3D photonic devices in the bulk of diamond with different geometries and at different depths, up to $200 \mu \mathrm{m}$ from the crystal surface. In terms of color center creation, femtosecond laser writing has established itself as a complementary method to ion beam implantation. The latter approach has the advantage of introducing foreign species into the diamond matrix to produce quantum emitters other than the $\mathrm{NV}^{-}$center, at the expenses of introducing substantial "collateral damage" in the diamond structure resulting in mechanical stresses that affect the opto-physical properties of the color centers. ${ }^{96,97}$ Although femtosecond laser writing has, thus, far demonstrated lower performance in terms of lateral and vertical placement accuracy, it offers superior capabilities to ion implantation in terms of vertical depth range on color center fabrication, limited only by the working distance of the focusing lens. Furthermore, the low ratio of quantum-optically functional color centers per implanted ion is still one of the main obstacles for the ion beam implantation technique, making it largely unsuitable for the scalable fabrication of multiple-qubit devices, while in the case of laser induced creation of $\mathrm{NV}^{-}$centers, unitary yield formation can be achieved. Overall, femtosecond laser writing in diamond has proven 
to be a powerful and versatile technique, allowing the implementation of integrated photonic circuits for both quantum information and sensing applications, through a simple and versatile setup. It has been demonstrated how $\mathrm{NV}^{-}$centers can be created and combined with the guiding properties of optical waveguides to develop room temperature devices with unprecedented spatial resolution and sensitivities. In Perspective, the research related to the femtosecond laser writing in diamond is still in an early stage, and as already shown, the possible developments include the optimization of the existing devices to the implementation of more complex systems, which, in the future, will play a key role in several technological fields.

A. N. Giakoumaki and G. Coccia are grateful for the support from the H2020 Marie Curie ITN project PHOTOTRAIN (Grant No.722591) and Lombardy Region Project sPATIALS3 "Miglioramento delle produzioni agroalimentari e tecnologie innovative per un 'alimentazione più sana, sicura e sostenibile," cofunded by POR FESR 2014-2020 Call HUB Ricerca e Innovazione. IFN-CNR, Ulm University, University of Torino, and Cardiff University are thankful for the financial support from the H2020 Marie Curie ITN project LasIonDef (Grant No. 956387). V. Bharadwaj is grateful for the support from the Alexander Von Humboldt Foundation.

\section{AUTHOR DECLARATIONS \\ Conflict of Interest}

The authors have no conflicts to disclose.

\section{Author Contributions}

A.N.G. and G.C. contributed equally to this work.

\section{DATA AVAILABILITY}

The data that support the findings of this study are available from the corresponding author upon reasonable request.

\section{REFERENCES}

${ }^{1}$ G. Balasubramanian, P. Neumann, D. Twitchen, M. Markham, R. Kolesov, N. Mizuochi, J. Isoya, J. Achard, J. Beck, J. Tissler, V. Jacques, P. R. Hemmer, F. Jelezko, and J. Wrachtrup, "Ultralong spin coherence time in isotopically engineered diamond," Nat. Mater. 8, 383 (2009).

${ }^{2}$ I. Aharonovich, A. D. Greentree, and S. Prawer, "Diamond photonics," Nat. Photonics 5, 397 (2011).

${ }^{3}$ F. Barry, M. J. Turner, J. M. Schloss, D. R. Glenn, Y. Song, M. D. Lukin, H. Park, J. F. Barry, M. J. Turner, J. M. Schloss, D. R. Glenn, Y. Song, and M. D. Lukin, "Optical magnetic detection of single-neuron action potentials using quantum defects in diamond," Proc. Natl. Acad. Sci. U. S. A. 114, 14133 (2017)

${ }^{4}$ M. W. Doherty, V. V. Struzhkin, D. A. Simpson, L. P. McGuinness, Y. Meng, A. Stacey, T. J. Karle, R. J. Hemley, N. B. Manson, L. C. L. Hollenberg, and S. Prawer, "Electronic properties and metrology applications of the diamond $\mathrm{NV}^{-}$center under pressure," Phys. Rev. Lett. 112, 47601 (2014).

${ }^{5}$ T. Plakhotnik, M. W. Doherty, J. H. Cole, R. Chapman, and N. B. Manson, "All-optical thermometry and thermal properties of the optically detected spin resonances of the $\mathrm{NV}^{-}$center in nanodiamond," Nano Lett. 14, 4989 (2014).

${ }^{6}$ J. R. Maze, P. L. Stanwix, J. S. Hodges, S. Hong, J. M. Taylor, P. Cappellaro, L. Jiang, M. V. G. Dutt, E. Togan, A. S. Zibrov, A. Yacoby, R. L. Walsworth, and M. D. Lukin, "Nanoscale magnetic sensing with an individual electronic spin in diamond," Nature 455, 644 (2008).

${ }^{7}$ A. Ajoy, U. Bissbort, M. D. Lukin, R. L. Walsworth, and P. Cappellaro, "Atomic-scale nuclear spin imaging using quantum-assisted sensors in diamond," Phys. Rev. X 5, 11001 (2015)
${ }^{8}$ G. Balasubramanian, I. Y. Chan, R. Kolesov, M. Al-Hmoud, J. Tisler, C. Shin, C. Kim, A. Wojcik, P. R. Hemmer, A. Krueger, T. Hanke, A. Leitenstorfer, R. Bratschitsch, F. Jelezko, and J. Wrachtrup, "Nanoscale imaging magnetometry with diamond spins under ambient conditions," Nature 455, 648 (2008).

${ }^{9}$ S. Kolkowitz, A. Safira, A. A. High, R. C. Devlin, S. Choi, Q. P. Unterreithmeier, D. Patterson, A. S. Zibrov, V. E. Manucharyan, H. Park, and M. D. Lukin, "Probing Johnson noise and ballistic transport in normal metals with a single-spin qubit," Science 347, 1129 (2015).

${ }^{10}$ G. Kucsko, P. C. Maurer, N. Y. Yao, M. Kubo, H. J. Noh, P. K. Lo, H. Park, and M. D. Lukin, "Nanometre-scale thermometry in a living cell," Nature 500, 54 (2013).

${ }^{11}$ B. B. Zhou, P. C. Jerger, K. H. Lee, M. Fukami, F. Mujid, J. Park, and D. D. Awschalom, "Spatiotemporal mapping of a photocurrent vortex in monolayer $\mathrm{MoS}_{2}$ using diamond quantum sensors," Phys. Rev. X 10, 11003 (2020).

${ }^{12}$ S. E. Lillie, N. Dontschuk, D. A. Broadway, D. L. Creedon, L. C. L. Hollenberg, and J. P. Tetienne, "Imaging graphene field-effect transistors on diamond using nitrogen-vacancy microscopy," Phys. Rev. Appl. 12, 24018 (2019).

${ }^{13}$ B. Khanaliloo, H. Jayakumar, A. C. Hryciw, D. P. Lake, H. Kaviani, and P. E. Barclay, "Single-crystal diamond nanobeam waveguide optomechanics," Phys. Rev. X 5, 41051 (2015).

${ }^{14}$ M. J. Burek, N. P. De Leon, B. J. Shields, B. J. M. Hausmann, Y. Chu, Q. Quan, A. S. Zibrov, H. Park, M. D. Lukin, and M. Lončar, "Free-standing mechanical and photonic nanostructures in single-crystal diamond," Nano Lett. 12, 6084 (2012).

${ }^{15}$ S. Lagomarsino, P. Olivero, F. Bosia, M. Vannoni, S. Calusi, L. Giuntini, and M. Massi, "Evidence of light guiding in ion-implanted diamond," Phys. Rev. Lett. 105, 233903 (2010).

${ }^{16}$ P. Olivero, S. Rubanov, P. Reichart, B. C. Gibson, S. T. Huntington, J. Rabeau, A. D. Greentree, J. Salzman, D. Moore, D. N. Jamieson, and S. Prawer, "Ionbeam-assisted lift-off technique for three-dimensional micromachining of freestanding single-crystal diamond," Adv. Mater. 17, 2427 (2005).

${ }^{17}$ B. Sotillo, V. Bharadwaj, J. P. Hadden, M. Sakakura, A. Chiappini, T. T. Fernandez, S. Longhi, O. Jedrkiewicz, Y. Shimotsuma, L. Criante, R. Osellame, G. Galzerano, M. Ferrari, K. Miura, R. Ramponi, P. E. Barclay, and S. M. Eaton, "Diamond photonics platform enabled by femtosecond laser writing," Sci. Rep. 6, 35566 (2016).

${ }^{18}$ A. Courvoisier, M. J. Booth, and P. S. Salter, "Inscription of 3D waveguides in diamond using an ultrafast laser,” Appl. Phys. Lett. 109, 031109 (2016).

${ }^{19}$ C. B. Schaffer, A. Brodeur, and E. Mazur, "Laser-induced breakdown and damage in bulk transparent materials induced by tightly focused femtosecond laser pulses," Meas. Sci. Technol. 12, 1784 (2001).

${ }^{20}$ D. Du, X. Liu, G. Korn, J. Squier, and G. Mourou, "Laser-induced breakdown by impact ionization in $\mathrm{SiO}_{2}$ with pulse widths from 7 ns to $150 \mathrm{fs}$," Appl. Phys. Lett. 64, 3071 (1994).

${ }^{21}$ V. Bharadwaj, A. Courvoisier, T. T. Fernandez, R. Ramponi, G. Galzerano, J. Nunn, M. J. Booth, R. Sellame, S. M. Eaton, and P. S. Salter, "Femtosecond laser inscription of Bragg grating waveguides in bulk diamond," Opt. Lett. 42, 3451 (2017).

${ }^{22}$ A. Abdelmalek, A. N. Giakoumaki, V. Bharadwaj, B. Sotillo, T. L. Phu, M. Bollani, Z. Bedrane, R. Ramponi, S. M. Eaton, and M. Maaza, "Morphological study of nanostructures induced by direct femtosecond laser ablation on diamond," Micromachines 12, 583 (2021).

${ }^{23}$ V. Bharadwaj, O. Jedrkiewicz, J. P. Hadden, B. Sotillo, M. R. Vázquez, P. Dentella, T. T. Fernandez, A. Chiappini, A. N. Giakoumaki, T. L. Phu, M. Bollani, M. Ferrari, R. Ramponi, P. E. Barclay, and S. M. Eaton, "Femtosecond laser written photonic and microfluidic circuits in diamond," J. Phys. 1, 22001 (2019).

${ }^{24}$ B. Sotillo, V. Bharadwaj, J. P. Hadden, S. Rampini, A. Chiappini, T. T. Fernandez, C. Armellini, A. Serpengüzel, M. Ferrari, P. E. Barclay, R. Ramponi, and S. M. Eaton, "Visible to infrared diamond photonics enabled by focused femtosecond laser pulses," Micromachines 8, 60 (2017).

${ }^{25}$ V. Bharadwaj, Y. Wang, T. T. Fernandez, R. Ramponi, S. M. Eaton, and G. Galzerano, "Femtosecond laser written diamond waveguides: A step towards integrated photonics in the far infrared," Opt. Mater. 85, 183-185 (2018).

${ }^{26}$ Y. C. Chen, P. S. Salter, S. Knauer, L. Weng, A. C. Frangeskou, C. J. Stephen, S. N. Ishmael, P. R. Dolan, S. Johnson, B. L. Green, G. W. Morley, M. E. Newton, 
J. G. Rarity, M. J. Booth, and J. M. Smith, "Laser writing of coherent colour centres in diamond," Nat. Photonics 11, 77 (2017).

${ }^{27}$ S. M. Eaton, J. P. Hadden, V. Bharadwaj, J. Forneris, F. Picollo, F. Bosia, B. Sotillo, A. N. Giakoumaki, O. Jedrkiewicz, A. Chiappini, M. Ferrari, R. Osellame, P. E. Barclay, P. Olivero, and R. Ramponi, "Quantum micro-nano devices fabricated in diamond by femtosecond laser and ion irradiation," Adv. Quantum Technol. 2, 1900006 (2019).

${ }^{28}$ M. P. Hiscocks, C. J. Kaalund, F. Ladouceur, S. T. Huntington, B. C. Gibson, S. Trpkovski, D. Simpson, E. Ampem-Lassen, and S. Prawer, "Diamond waveguides: Toward an all-diamond platform," Proc. SPIE 6801, 68010J (2008).

${ }^{29}$ M. P. Hiscocks, K. Ganesan, B. C. Gibson, S. T. Huntington, F. Ladouceur, and S. Prawer, "Diamond waveguides fabricated by reactive ion etching," Opt. Express 16, 19512-19519 (2008).

${ }^{30}$ T. Schröder, S. L. Mouradian, J. Zheng, M. E. Trusheim, M. Walsh, E. H. Chen, L. Li, I. Bayn, and D. Englund, "Quantum nanophotonics in diamond," J. Opt. Soc. Am. B 33, B65-B83 (2016).

${ }^{31}$ J. R. Rabeau, P. Reichart, G. Tamanyan, D. N. Jamieson, S. Prawer, F. Jelezko, and J. Wrachtrup, "Implantation of labelled single nitrogen vacancy centers in diamond using $\mathrm{N}^{15}$," Appl. Phys. Lett. 88(2), 023113 (2006).

${ }^{32}$ C. Santori, P. E. Barclay, K. M. C. Fu, and R. G. Beausoleil, "Vertical distribution of nitrogen-vacancy centers in diamond formed by ion implantation and annealing," Phys. Rev. B 79(12), 125313 (2009).

${ }^{33}$ J. O. Orwa, C. Santori, K. M. C. Fu, B. Gibson, D. Simpson, I. Aharonovich, A. Stacey, A. Cimmino, P. Balog, M. Markham, D. Twitchen, A. D. Greentree, R. G. Beausoleil, and S. Prawer, "Engineering of nitrogen-vacancy color centers in high purity diamond by ion implantation and annealing," J. Appl. Phys. 109, 83530 (2011).

${ }^{34}$ C. Bradac, W. Gao, J. Forneris, M. E. Trusheim, and I. Aharonovich, "Quantum nanophotonics with group IV defects in diamond," Nat. Commun, 10(1), 1-13 (2019).

${ }^{35}$ K. M. Davis, K. Miura, N. Sugimoto, and K. Hirao, "Writing waveguides in glass with a femtosecond laser," Opt. Lett. 21, 1729 (1996).

${ }^{36}$ S. M. Eaton, H. Zhang, P. R. Herman, F. Yoshino, L. Shah, J. Bovatsek, and A. Y. Arai, "Heat accumulation effects in femtosecond laser-written waveguides with variable repetition rate," Opt. Express 13, 4708 (2005).

${ }^{37}$ R. R. Gattass and E. Mazur, "Femtosecond laser micromachining in transparent materials," Nat. Photonics 2, 219 (2008).

${ }^{38} \mathrm{~F}$. Chen and J. R. V. de Aldana, "Optical waveguides in crystalline dielectric materials produced by femtosecond-laser micromachining," Laser Photonics Rev. 8, 251 (2014)

${ }^{39}$ B. Sotillo, A. Chiappini, V. Bharadwaj, J. P. Hadden, F. Bosia, P. Olivero, M. Ferrari, R. Ramponi, P. E. Barclay, and S. M. Eaton, "Polarized micro-Raman studies of femtosecond laser written stress-induced optical waveguides in diamond," Appl. Phys. Lett. 112, 031109 (2018).

${ }^{40} \mathrm{R}$. M. Waxler and C. E. Weir, "Effect of hydrostatic pressure on the refractive indices of some solids," J. Res. Natl, Bur. Stand., Sect. A 69A, 325 (1965).

${ }^{41}$ J. Fontanella, R. L. Johnston, J. H. Colwell, and C. Andeen, "Temperature and pressure variation of the refractive index of diamond," Appl. Opt. 16, 2949 (1977).

${ }^{42}$ N. M. Balzaretti and J. A. H. Da Jornada, "Pressure dependence of the refractive index of diamond, cubic silicon carbide and cubic boron nitride," Solid State Commun. 99, 943 (1996).

${ }^{43}$ F. Bosia, N. Argiolas, M. Bazzan, B. A. Fairchild, A. D. Greentree, D. W. M. Lau, P. Olivero, F. Picollo, S. Rubanov, and S. Prawer, "Direct measurement and modelling of internal strains in ion-implanted diamond," J. Phys: Condens. Matter 25, 385403 (2013).

${ }^{44}$ G. García, I. Preda, M. Díaz-Híjar, V. Tormo-Márquez, O. Peña-Rodríguez, J. Olivares, F. Bosia, N. M. Pugno, F. Picollo, L. Giuntini, A. Sordini, P. Olivero, L. López-Mir, and C. Ocal, "Micro and nano-patterning of single-crystal diamond by swift heavy ion irradiation," Diamond Relat. Mater. 69, 1-7 (2016).

${ }^{45}$ Y. Liu, G. Chen, M. Song, X. Ci, B. Wu, E. Wu, and H. Zeng, "Fabrication of nitrogen vacancy color centers by femtosecond pulse laser illumination," Opt. Express 21, 12843 (2013).

${ }^{46}$ V. Yurgens, J. A. Zuber, S. Fla ${ }^{\circ}$ gan, M. De Luca, B. J. Shields, I. Zardo, P. Maletinsky, J. W. RIchard, and T. Jakubczyk, "Low-charge-noise nitrogenvacancy centers in diamond created using laser writing with a solid-immersion lens," ACS Photonics 8, 1726-1734 (2021).
${ }^{47}$ S. Lagomarsino, A. M. Flatae, S. Sciortino, F. Gorelli, M. Santoro, F. Tantussi, F. De Angelis, N. Gelli, F. Taccetti, L. Giuntini, and M. Agio, "Optical properties of silicon-vacancy color centers in diamond created by ion implantation and post-annealing," Diamond Relat. Mater. 84, 196-203 (2018).

${ }^{48}$ H. J. Kimble, "The quantum internet," Nature 453, 1023 (2008).

${ }^{49}$ Q. Shi, B. Sontheimer, N. Nikolay, A. W. Schell, J. Fischer, A. Naber, O. Benson, and M. Wegener, "Wiring up pre-characterized single-photon emitters by laser lithography," Sci. Rep. 6, 31135 (2016).

${ }^{50}$ A. Sipahigil, R. E. Evans, D. D. Sukachev, M. J. Burek, J. Borregaard, M. K. Bhaskar, C. T. Nguyen, J. L. Pacheco, H. A. Atikian, C. Meuwly, R. M. Camacho, F. Jelezko, E. Bielejec, H. Park, M. Lončar, and M. D. Lukin, "An integrated diamond nanophotonics platform for quantum-optical networks," Science 354, 847 (2016).

${ }^{51}$ J. P. Hadden, V. Bharadwaj, B. Sotillo, S. Rampini, R. Osellame, J. D. Witmer, H. Jayakumar, T. T. Fernandez, A. Chiappini, C. Armellini, M. Ferrari, R. Ramponi, P. E. Barclay, and S. M. Eaton, "Integrated waveguides and deterministically positioned nitrogen vacancy centers in diamond created by femtosecond laser writing," Opt. Lett. 43, 3586-3589 (2018).

${ }^{52}$ B. Naydenov, F. Reinhard, A. Lämmle, V. Richter, R. Kalish, U. F. S. D'Haenens-Johansson, M. Newton, F. Jelezko, and J. Wrachtrup, "Increasing the coherence time of single electron spins in diamond by high temperature annealing," Appl. Phys. Lett. 97, 242511 (2010).

${ }^{53}$ A. Benayas, D. Jaque, B. McMillen, and K. P. Chen, "Ultrafast laser writing of optical waveguides in ceramic Yb: YAG: A study of thermal and non-thermal regimes," J. Appl. Phys 104, 301 (2011).

${ }^{54}$ M. W. Doherty, F. Dolde, H. Fedder, F. Jelezko, J. Wrachtrup, N. B. Manson, and L. C. L. Hollenberg, "Theory of the ground-state spin of the $\mathrm{NV}^{-}$center in diamond," Phys. Rev. B 85, 205203 (2012).

${ }^{55}$ F. Dolde, H. Fedder, M. W. Doherty, T. Nöbauer, F. Rempp, G. Balasubramanian, T. Wolf, F. Reinhard, L. C. L. Hollenberg, F. Jelezko, and J. Wrachtrup, "Electricfield sensing using single diamond spins," Nat. Phys. 7, 459 (2011).

${ }^{56}$ J. M. Taylor, P. Cappellaro, L. Childress, L. Jiang, D. Budker, P. R. Hemmer, A. Yacoby, R. Walsworth, and M. D. Lukin, "High-sensitivity diamond magnetometer with nanoscale resolution," Nat. Phys. 4, 810 (2008).

${ }^{57}$ F. Dolde, M. W. Doherty, J. Michl, I. Jakobi, B. Naydenov, S. Pezzagna, J. Meijer, P. Neumann, F. Jelezko, N. B. Manson, and J. Wrachtrup, "Nanoscale detection of a single fundamental charge in ambient conditions using the $\mathrm{NV}^{-}$ center in diamond," Phys. Rev. Lett. 112, 97603 (2014).

${ }^{58}$ A. Dréau, M. Lesik, L. Rondin, P. Spinicelli, O. Arcizet, J. F. Roch, and V. Jacques, "Avoiding power broadening in optically detected magnetic resonance of single NV defects for enhanced dc magnetic field sensitivity," Phys. Rev. B 84, 195204 (2011).

${ }^{59}$ E. H. Chen, H. A. Clevenson, K. A. Johnson, L. M. Pham, D. R. Englund, P. R. Hemmer, and D. A. Braje, "High-sensitivity spin-based electrometry with an ensemble of nitrogen-vacancy centers in diamond," Phys. Rev. A 95, 53417 (2017).

${ }^{60}$ L. M. Pham, D. L. Sage, P. L. Stanwix, T. K. Yeung, D. Glenn, A. Trifonov, P. Cappellaro, P. R. Hemmer, M. D. Lukin, H. Park, A. Yacoby, and R. L. Walsworth, "Magnetic field imaging with nitrogen-vacancy ensembles," New J. Phys. 13, 045021 (2011).

${ }^{61}$ D. L. Sage, L. M. Pham, N. Bar-Gill, C. Belthangady, M. D. Lukin, A. Yacoby, and R. L. Walsworth, "Efficient photon detection from color centers in a diamond optical waveguide," Phys. Rev. B 85, 121202 (2012).

${ }^{62}$ I. Aharonovich, C. Santori, B. A. Fairchild, J. Orwa, K. Ganesan, K. M. C. Fu, R. G. Beausoleil, A. D. Greentree, and S. Prawer, "Producing optimized ensembles of nitrogen-vacancy color centers for quantum information applications," J. Appl. Phys. 106, 124904 (2009).

${ }^{63} \mathrm{H}$. Clevenson, M. E. Trusheim, C. Teale, T. Schröder, D. Braje, and D. Englund, "Broadband magnetometry and temperature sensing with a lighttrapping diamond waveguide," Nat. Phys. 11, 393 (2015).

${ }^{64}$ M. Hoese, M. K. Koch, V. Bharadwaj, J. Lang, J. P. Hadden, R. Yoshizaki, A. N. Giakoumaki, R. Ramponi, F. Jelezko, S. M. Eaton, and A. Kubanek, "Integrated magnetometry platform with stackable waveguide-assisted detection channels for sensing arrays," Phys. Rev. Appl. 15, 54059 (2021).

${ }^{65}$ F. Jelezko, T. Gaebel, I. Popa, A. Gruber, and J. Wrachtrup, "Observation of coherent oscillations in a single electron spin,” Phys. Rev. Lett. 92, 76401 (2004). 
${ }^{66} \mathrm{~B}$. Julsgaard and K. Molmer, "Dynamical evolution of an inverted spin ensemble in a cavity: Inhomogeneous broadening as a stabilizing mechanism," Phys. Rev. A 86, 63810 (2012).

${ }^{67}$ Y. Matsuzaki, H. Morishita, T. Shimooka, T. Tashima, K. Kakuyanagi, K. Semba, W. J. Munro, H. Yamaguchi, N. Mizuochi, and S. Saito, "Optically detected magnetic resonance of high-density ensemble of $\mathrm{NV}^{-}$centers in diamond," J. Phys.: Condens. Matter 28, 275302 (2016)

${ }^{68}$ A. Kuwahata, T. Kitaizumi, K. Saichi, T. Sato, R. Igarashi, T. Ohshima, Y. Masuyama, T. Iwasaki, M. Hatano, F. Jelezko, M. Kusakabe, T. Yatsui, and M. Sekino, "Magnetometer with nitrogen-vacancy center in a bulk diamond for detecting magnetic nanoparticles in biomedical applications," Sci. Rep. 10, 2483 (2020).

${ }^{69}$ E. Moreva, E. Bernardi, P. Traina, A. Sosso, S. Ditalia Tchernij, J. Forneris, F. Picollo, G. Brida, Ž. Pastuović, I. P. Degiovanni, P. Olivero, and M. Genovese, "Practical applications of quantum sensing: A simple method to enhance the sensitivity of nitrogen-vacancy-based temperature sensors," Phys. Rev. Appl. 13, 54057 (2020).

${ }^{70}$ E. V. Levine, M. J. Turner, P. Kehayias, C. A. Hart, N. Langellier, R. Trubko, D. R. Glenn, R. R. Fu, and R. L. Walsworth, "Principles and techniques of the quantum diamond microscope," Nanophotonics 8, 1945 (2019).

${ }^{77}$ F. Gorrini, C. Dorigoni, D. Olivares-Postigo, R. Giri, P. Aprà, F. Picollo, and A. Bifone, "Photoconversion of shallow nitrogen-vacancy centers in flat and nanotruncated diamond under high-power laser irradiation," arXiv:2105.04939 (2021).

${ }^{72}$ A. Sipahigil, K. D. Jahnke, L. J. Rogers, T. Teraji, J. Isoya, A. S. Zibrov, F. Jelezko, and M. D. Lukin, "Indistinguishable photons from separated siliconvacancy centers in diamond," Phys. Rev. Lett. 113, 113602 (2014).

${ }^{73}$ Y. Rong, K. Cheng, Z. Ju, C. Pan, Q. Ma, S. Liu, S. Shen, B. Wu, T. Jia, E. Wu, and H. Zeng, "Bright near-surface silicon vacancy centers in diamond fabricated by femtosecond laser ablation," Opt. Lett. 44, 3793-3796 (2019).

${ }^{74}$ J. Lang, S. Häußler, J. Fuhrmann, R. Waltrich, S. Laddha, J. Scharpf, A. Kubanek, B. Naydenov, and F. Jelezko, "Long optical coherence times of shallow-implanted, negatively charged silicon vacancy centers in diamond," Appl. Phys. Lett. 116, 064001 (2020).

${ }^{75}$ Y. N. Palyanov, I. N. Kupriyanov, Y. M. Borzdov, and N. V. Surovtsev, "Germanium: A new catalyst for diamond synthesis and a new optically active impurity in diamond," Sci. Rep. 5, 14789 (2015).

${ }^{76}$ E. A. Ekimov, S. G. Lyapin, and M. V. Kondrin, "Tin-vacancy color centers in micro-and polycrystalline diamonds synthesized at high pressures," Diamond Relat. Mater. 87, 223-227 (2018).

${ }^{77}$ T. Iwasaki, F. Ishibashi, Y. Miyamoto, Y. Doi, S. Kobayashi, T. Miyazaki, K. Tahara, K. D. Jahnke, L. J. Rogers, B. Naydenov, F. Jelezko, S. Yamasaki, S. Nagamachi, T. Inubushi, N. Mizuochi, and M. Hatano, "Germanium-vacancy single color centers in diamond,” Sci. Rep. 5, 12882 (2015).

${ }^{78}$ T. Iwasaki, Y. Miyamoto, T. Taniguchi, P. Siyushev, M. H. Metsch, F. Jelezko, and M. Hatano, "Tin-vacancy quantum emitters in diamond," Phys. Rev. Lett. 119, 253601 (2017).

${ }^{79}$ S. D. Tchernij, T. Herzig, J. Forneris, J. Kupper, S. Pezzagna, P. Traina, E. Moreva, I. P. Degiovanni, G. Brida, N. Skukan, M. Genovese, M. Jakšić, J. Meijer, and P. Olivero, "Single-photon-emitting optical centers in diamond fabricated upon Sn implantation," ACS Photonics 4, 2580-2586 (2017).

${ }^{80}$ P. Siyushev, M. H. Metsch, A. Ijaz, J. M. Binder, M. K. Bhaskar, D. D. Sukachev, A. Sipahigil, R. E. Evans, C. T. Nguyen, M. D. Lukin, P. R. Hemmer,
Y. N. Palyanov, I. N. Kupriyanov, Y. M. Borzdov, L. J. Rogers, and F. Jelezko, "Optical and microwave control of germanium-vacancy center spins in diamond," Phys. Rev. B 96, 081201 (2017).

${ }^{81}$ S. D. Tchernij, T. Lühmann, T. Herzig, J. Küpper, A. Damin, S. Santonocito, M. Signorile, P. Traina, E. Moreva, F. Celegato, S. Pezzagna, I. P. Degiovanni, P. Olivero, M. Jakšíc, J. Meijer, P. M. Genovese, and J. Forneris, J. ACS Photonics 5, 4864 (2018).

${ }^{82}$ X. Gao, S. Pandey, M. Kianinia, J. Ahn, P. Ju, I. Aharonovich, N. Shivaram, and $\mathrm{T}$. $\mathrm{Li}$, "Femtosecond laser writing of spin defects in hexagonal boron nitride," ACS Photonics 8, 994-1000 (2021).

${ }^{83}$ Y. C. Chen, P. S. Salter, M. Niethammer, M. Widmann, F. Kaiser, R. Nagy, N. Morioka, C. Babin, J. Erlekampf, P. Berwian, J. B. Martin, and J. Wrachtrup, "Laser writing of scalable single color centers in silicon carbide," Nano Lett. 19, 2377-2383 (2019).

${ }^{84}$ S. Gao, Y. Z. Duan, Z. N. Tian, Y. L. Zhang, Q. D. Chen, B. R. Gao, and H. B. Sun, "Laser-induced color centers in crystals," Opt. Laser Technol. 146, 107527 (2022).

${ }^{85}$ V. V. Belloni, M. Bollani, S. M. Eaton, P. D. Trapani, and O. Jedrkiewicz, "Micro-hole generation by high-energy pulsed bessel beams in different transparent materials," Micromachines 12, 455 (2021).

${ }^{86}$ R. Giri, F. Gorrini, C. Dorigoni, C. E. Avalos, M. Cazzanelli, S. Tambalo, and A. Bifone, "Coupled charge and spin dynamics in high-density ensembles of nitrogen-vacancy centers in diamond," Phys. Rev. B 98, 45401 (2018).

${ }^{87}$ F. Gorrini, R. Giri, C. E. Avalos, S. Tambalo, S. Mannucci, L. Basso, N. Bazzanella, C. Dorigoni, M. Cazzanelli, P. Marzola, A. Miotello, and A. Bifone, "Fast and sensitive detection of paramagnetic species using coupled charge and spin dynamics in strongly fluorescent nanodiamonds," ACS Appl. Mater. Interfaces 11, 24412 (2019).

${ }^{88}$ G. D. Marshall, A. Politi, J. C. F. Matthews, P. Dekker, M. Ams, M. J. Withford, and J. L. O’Brien, "Laser written waveguide photonic quantum circuits," Opt. Express 17, 12546 (2009).

${ }^{89}$ V. S. Vavilov, V. V. Krasnopevtsev, Y. V. Miljutin, A. E. Gorodetsky, and A. P. Zakharov, "On structural transitions in ion-implanted diamond," Radiat. Eff. 22, 141-143 (1974).

${ }^{90}$ Y. G. Chen, M. Hasegawa, H. Okushi, S. Koizum, H. Yoshida, T. Sakai, and N. Kobayashi, Diamond Relat. Mater. 11, 451 (2002).

${ }^{91}$ J. Forneris, P. Traina, D. Gatto Monticone, G. Amato, L. Boarino, G. Brida, I. P. Degiovanni, E. Enrico, E. Moreva, V. Grilj, N. Skukan, M. Jakšić, M. Genovese, and P. Olivero, Sci. Rep. 5, 15901 (2015).

${ }^{92}$ P. Olivero, J. Forneris, M. Jakšić, Ž. Pastuović, F. Picollo, N. Skukan, and E. Vittone, Nucl. Instrum. Methods Phys. Res., Sect. B 269, 2340-2344 (2011).

${ }^{93}$ M. Shimizu, Y. Shimotsuma, M. Sakakura, T. Yuasa, H. Homma, Y. Minowa, K. Tanaka, K. Miura, and K. Hirao, Opt. Express 17, 46 (2009).

${ }^{94}$ P. Olivero, S. Calusi, L. Giuntini, S. Lagomarsino, A. Lo Giudice, M. Massi, S. Sciortino, M. Vannoni, and E. Vittone, Diamond Relat. Mater. 19(5-6), 428-431 (2010).

${ }^{95}$ F. Picollo, A. Battiato, E. Bernardi, A. Marcantoni, A. Pasquarelli, E. Carbone, P. Olivero, and V. Carabelli, Anal. Chem. 88, 7493-7499 (2016).

${ }^{96}$ F. Bosia, P. Olivero, E. Vittone, F. Picollo, A. Lo Giudice, M. Jakšić, N. Skukan, L. Giuntini, M. Massi, S. Calusi, M. Vannoni, S. Lagomarsino, and S. Sciortino, Nucl. Instrum. Methods Phys. Res., Sect. B 268, 2991-2995 (2010).

${ }^{97}$ P. Olivero, F. Bosia, B. A. Fairchild, B. C. Gibson, A. D. Greentree, P. Spizzirri, and S. Prawer, New J. Phys. 15, 043027 (2013). 